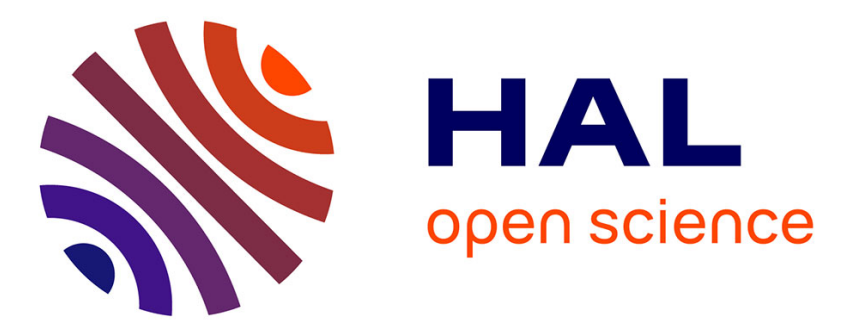

\title{
New advances in the forced response computation of periodic structures using the wave finite element (WFE) method \\ Jean-Mathieu Mencik
}

\section{- To cite this version:}

Jean-Mathieu Mencik. New advances in the forced response computation of periodic structures using the wave finite element (WFE) method. Computational Mechanics, 2014, 54 (3), pp.789-801. 10.1007/s00466-014-1033-1 . hal-01056149

\section{HAL Id: hal-01056149 \\ https://hal.science/hal-01056149}

Submitted on 16 Aug 2014

HAL is a multi-disciplinary open access archive for the deposit and dissemination of scientific research documents, whether they are published or not. The documents may come from teaching and research institutions in France or abroad, or from public or private research centers.
L'archive ouverte pluridisciplinaire HAL, est destinée au dépôt et à la diffusion de documents scientifiques de niveau recherche, publiés ou non, émanant des établissements d'enseignement et de recherche français ou étrangers, des laboratoires publics ou privés. 


\title{
New advances in the forced response computation of periodic structures using the wave finite element (WFE) method
}

\author{
J.-M. Mencik
}

\begin{abstract}
The wave finite element (WFE) method is investigated to describe the harmonic forced response of onedimensional periodic structures like those composed of complex substructures and encountered in engineering applications. The dynamic behavior of these periodic structures is analyzed over wide frequency bands where complex spatial dynamics, inside the substructures, are likely to occur. Within the WFE framework, the dynamic behavior of periodic structures is described in terms of numerical wave modes. Their computation follows from the consideration of the finite element model of a substructure that involves a large number of internal degrees of freedom. Some rules of thumb of the WFE method are highlighted and discussed to circumvent numerical issues like ill-conditioning and instabilities. It is shown for instance that an exact analytic relation needs to be considered to enforce the coherence between positive-going and negative-going wave modes. Besides, a strategy is proposed to interpolate the frequency response functions of periodic structures at a reduced number of discrete frequencies. This strategy is proposed to tackle the problem of large CPU times involved when the wave modes are to be computed many times. An error indicator is formulated which provides a good estimation of the level of accuracy of the interpolated solutions at intermediate points. Adaptive refinement is carried out to ensure that this error indicator remains below a certain tolerance threshold. $\mathrm{Nu}$ merical experiments highlight the relevance of the proposed approaches.
\end{abstract}

Keywords structural dynamics; wave finite element method; periodic structures; model reduction.

J.-M. Mencik

INSA Centre Val de Loire, Université François Rabelais de Tours, LMR EA 2640, Campus de Blois, 3 rue de la chocolaterie, CS 23410, 41034 Blois Cedex, France

E-mail: jean-mathieu.mencik@insa-cvl.fr

\section{Introduction}

Predicting the frequency behavior of one-dimensional periodic structures, by means of efficient and fast numerical tools, is an open industrial challenge. Popular examples of those structures are aircraft fuselages, consisting of cylindrical panels with a periodic distribution of stiffeners, or parts of chassis frames with a periodic inclusion of holes. Usually, the number of degrees of freedom (DOFs) used to model each substructure - i.e., each periodic cell is large, which makes the conventional finite element (FE) method time consuming to assess the dynamic behavior of a whole periodic structure. This issue is addressed here within the framework of the wave finite element (WFE) method.

Originally, the WFE method has been initiated to describe the wave propagation along one-dimensional periodic waveguides, i.e. systems that are composed of identical substructures along one main direction [12,23,6,9]. For any waveguide, numerical wave modes are calculated by solving an eigenproblem which follows from the consideration of the FE model of one substructure. The wave modes are to be understood as particular solutions of the displacement/rotation and force/moment fields, at the interfaces between substructures, which travel from substructure to substructure along the waveguide. In fact, there are twice as many wave modes as the number of DOFs used to discretize each substructure boundary. Increasing that number of DOFs makes the WFE method efficient to assess the complex wave motions occurring as the frequency grows.

Previous works made on the WFE topic have been carried out to assess the guided wave propagation along straight elastic waveguides [23,15], fluid-filled pipes [16,11] and truss beams [20]. Besides, the WFE method has been applied to describe the plane wave propagation in infinite twodimensional structures [10]. Other wave-based numerical approaches have been developed over the past years. Among these is the semi-analytical finite element (SAFE) method, 
which has been applied to describe the guided wave propagation along elastic and elasto-acoustic straight waveguides [5,17]. In [8], a so-called reduced Bloch mode expansion (RBME) method has been proposed for fast band structure calculations in infinite periodic media. The RBME method is based on a unit cell domain extending in the direction of the wave propagation. One of the features of the RBME method is the utilization of a set of basis functions using unit cell symmetry properties in order to obtain a reduced model for a periodic medium as a whole, as represented by the unit cell. Although elegant, the RBME method remains however confined to the study of infinite periodic systems, while the SAFE method appears to be limited to the study of straight waveguides.

The issue of computing the forced response of straight elastic waveguides, by means of the WFE method, has been investigated in several ways $[13,21,18,4]$. In [13], an analytical relation has been proposed (see also $[6,9]$ ) which strictly enforces the coherence between wave modes traveling in positive and negative directions. Without that relation, the accuracy of the WFE method cannot be guaranteed as numerical dispersion and instabilities are likely to occur. The drawback of this analytical relation, however, is that the substructures are required to be symmetric with respect to their mid-plane.

The remarkable feature of the WFE method is that it enables a large decrease of the CPU times in comparison with the conventional FE method. This is explained since wave-based matrix systems of small size — which relates the number of DOFs on the cross-section of waveguides only - are considered rather than the full FE models of the waveguides. The WFE method has proved to be relevant, compared to the FE method and the component mode synthesis technique, to describe the dynamic behavior of sophisticated structures (see [14]).

So far, the WFE method has not yet been applied to the study of bounded periodic structures that involve complex substructures, i.e., whose FE models contain many internal DOFs. The underlying issue is that a dynamic condensation procedure is to be considered prior to the computation of wave modes, which may impact the conditioning of the wave-based matrix systems. The consideration of substructures having large size FE models appears also questionable because the computation of wave modes, at many discrete frequencies, may be time consuming. Interpolation techniques of frequency response functions (FRFs) seem to constitute interesting solutions to address this issue. In this framework, the vectors of displacements/rotations of a structure are explicitly computed at a reduced number of discrete frequencies - namely, the interpolation points while they are approximated at several intermediate points. Adaptive refinement of the sampling of interpolation points is achieved when the error, between the exact and approx- imated solutions, exceeds a specified tolerance threshold at any intermediate point. The issue here can be viewed as formulating an error indicator, given that the true error is not explicitly known. Such an issue is not new as already treated in various ways to address FRFs issued from matrix systems that are either linear or quadratic with respect to the frequency $[22,19,7]$. However, these procedures appear inefficient to address the FRFs issued from complex matrix systems like those involved by the WFE method, which are neither linear nor quadratic. In other words, it seems that the development of an interpolation technique, capable of handling the FRFs issued from the WFE method, has not been carried out yet.

To summarize, the motivation behind the present work appears as follows: (i) to prove that the WFE method can compete with the conventional FE method to describe the wide band frequency behavior of sophisticated periodic structures; (ii) to develop, within the WFE framework, an interpolation strategy that speeds up the computation of the FRFs.

The rest of the paper is organized as follows. In Section 2, the basics of the WFE method are recalled regarding the computation of wave modes and the forced response of periodic structures. Some rules of thumb of the method are presented and discussed. One constraint, outlined here, is that the substructures are to be symmetric with respect to their mid-plane. It is shown, however, that the FE meshes of the substructures do not need to be symmetric as long as the mesh density is high enough. In Section 3, a technique is proposed to interpolate the WFE-based FRFs at a reduced number of discrete frequencies. The technique uses adaptive refinement, which is achieved by assessing the accuracy of the interpolated solutions at intermediate points. An error indicator is formulated which can be evaluated quickly without the need of explicitly computing the wave modes. Numerical experiments are carried out in Section 4 to highlight the relevance of the proposed WFE strategies. The following test cases are considered: a beam with holes; a curved stiffened panel.

\section{WFE method}

\subsection{Concept of wave modes}

\subsubsection{Theory}

The WFE method aims at describing the one-dimensional wave propagation along periodic structures, i.e. elastic systems which, in the present framework, are composed of identical substructures along one straight direction (Fig. 1). Those elastic systems are assumed to be dissipative, with a loss 
factor $\eta$, and subject to harmonic disturbances at an angular frequency $\omega$. The proposed study is confined to the study of linear structures undergoing small strains, small displacements and small rotations; deformation modes that may develop due to kinematic nonlinearities are not taken into account. The description of waves traveling along a periodic structure is achieved by considering the FE model of one substructure (Fig. 2). The procedure is widely explained in the literature (see e.g. [23,15]); for the sake of clarity, it is summarized hereafter.

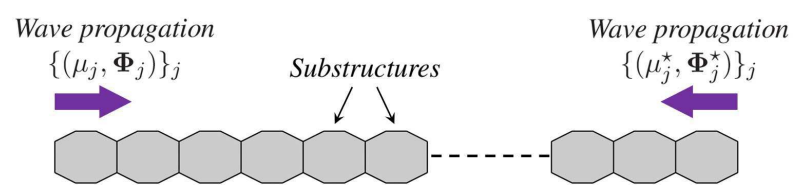

Fig. 1 Illustration of a periodic structure.

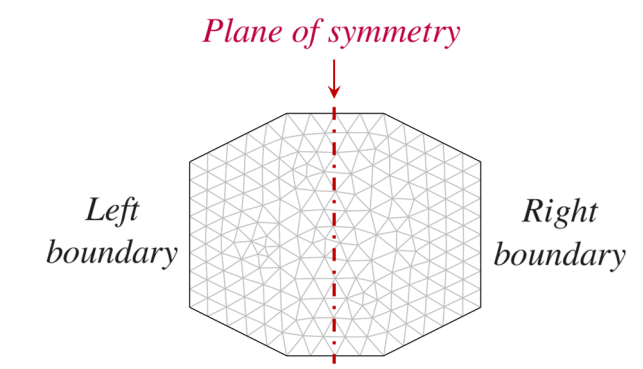

Fig. 2 FE model of a substructure.

A state vector representation is considered to express the kinematic/mechanical quantities - i.e., displacements, rotations, forces and moments - on the right boundary of the substructure from those on the left boundary (Fig. 2). In this framework, the left and right boundaries are discretized with a same number $n$ of DOFs. The substructure is modeled by means of a dynamic stiffness matrix, defined as $\mathbf{D}=$ $-\omega^{2} \mathbf{M}+(1+\mathbf{i} \eta) \mathbf{K}$ ( $\mathbf{M}$ and $\mathbf{K}$ being the mass and stiffness matrices of the substructure, respectively). The state vector representation is given by

$\mathbf{u}_{\mathrm{R}}=\mathbf{S u}_{\mathrm{L}}$

where the subscripts $L$ and $R$ denote the DOFs contained on the left and right boundaries of the substructure, respectively. Also, $\mathbf{u}_{\mathrm{R}}$ and $\mathbf{u}_{\mathrm{L}}$ are $2 n \times 1$ vectors, expressed as

$\mathbf{u}_{\mathrm{R}}=\left[\begin{array}{c}\mathbf{q}_{\mathrm{R}} \\ \mathbf{F}_{\mathrm{R}}\end{array}\right] \quad, \quad \mathbf{u}_{\mathrm{L}}=\left[\begin{array}{c}\mathbf{q}_{\mathrm{L}} \\ -\mathbf{F}_{\mathrm{L}}\end{array}\right]$, where $\mathbf{q}_{\mathrm{R}}$ and $\mathbf{F}_{\mathrm{R}}$ (resp. $\mathbf{q}_{\mathrm{L}}$ and $\mathbf{F}_{\mathrm{L}}$ ) are $n \times 1$ vectors of displacements/rotations and forces/moments, on the right (resp. left) boundary of the substructure. In Eq. (1), $\mathbf{S}$ is a $2 n \times 2 n$ matrix expressed as

$\mathbf{S}=\left[\begin{array}{c|c}-\mathbf{D}_{\mathrm{LR}}^{*-1} \mathbf{D}_{\mathrm{LL}}^{*} & -\mathbf{D}_{\mathrm{LR}}^{*-1} \\ \hline \mathbf{D}_{\mathrm{RL}}^{*}-\mathbf{D}_{\mathrm{RR}}^{*} \mathbf{D}_{\mathrm{LR}}^{*-1} \mathbf{D}_{\mathrm{LL}}^{*} & -\mathbf{D}_{\mathrm{RR}}^{*} \mathbf{D}_{\mathrm{LR}}^{*-1}\end{array}\right]$,

where $\mathbf{D}^{*}$ is the dynamic stiffness matrix of the substructure, condensed on its left and right boundaries. It is expressed as

$\mathbf{D}^{*}=\mathbf{D}_{\mathrm{BB}}-\mathbf{D}_{\mathrm{BI}} \mathbf{D}_{\mathrm{II}}^{-1} \mathbf{D}_{\mathrm{IB}}$,

where the subscripts B and I refer to the boundary DOFs (i.e., those contained on the left and right boundaries) and the internal DOFs, respectively. In particular, the matrix $\mathbf{D}_{\mathrm{BB}}$ is expressed as

$\mathbf{D}_{\mathrm{BB}}=\left[\begin{array}{ll}\mathbf{D}_{\mathrm{LL}} & \mathbf{D}_{\mathrm{LR}} \\ \mathbf{D}_{\mathrm{RL}} & \mathbf{D}_{\mathrm{RR}}\end{array}\right]$.

Also, the matrix $\mathbf{S}$ is symplectic [23], which means that $\mathbf{S}^{T} \mathbf{J S}=\mathbf{J}$, where

$\mathbf{J}=\left[\begin{array}{cc}\mathbf{0} & \mathbf{I}_{n} \\ -\mathbf{I}_{n} & \mathbf{0}\end{array}\right]$.

Besides, the coupling conditions at the interface between two consecutive substructures, labeled as $k-1$ and $k$, are expressed as $\mathbf{u}_{\mathrm{R}}^{(k-1)}=\mathbf{u}_{\mathrm{L}}^{(k)}$, i.e., the displacements/rotations are continuous across the interface, while the forces/moments satisfy the action-reaction law. As a result, Eq. (1) leads to

$\mathbf{u}_{\mathrm{L}}^{(k)}=\mathbf{S u}_{\mathrm{L}}^{(k-1)}$.

Following the WFE framework, the so-called state vectors $\mathbf{u}_{\mathrm{L}}^{(k-1)}$ and $\mathbf{u}_{\mathrm{L}}^{(k)}$ are expressed as $\mathbf{u}_{\mathrm{L}}^{(k-1)}=\sum_{j} Q_{j}^{(k-1)} \phi_{j}$ and $\mathbf{u}_{\mathrm{L}}^{(k)}=\sum_{j} Q_{j}^{(k)} \phi_{j}$, where $\left\{\boldsymbol{\phi}_{j}\right\}_{j}$ are the right eigenvectors of $\mathbf{S}$, while $\left\{Q_{j}\right\}_{j}$ play the role of amplitudes. Also, the eigenvalues of $\mathbf{S}$ are denoted as $\left\{\mu_{j}\right\}_{j}$. In fact, since the structure is periodic, it is readily verified that each amplitude $Q_{j}$ varies as $Q_{j}^{(k)}=\mu_{j}^{h} Q_{j}^{(k-h)}$, between one substructure $k-h$ to one substructure $k(0 \leq h \leq k-1)$ [13]. The physical meaning of the eigenvectors and eigenvalues of $\mathbf{S}$ follows from Bloch's theorem, where it is stated that $\phi_{j}$ represents a wave shape traveling along the waveguide as $\mu_{j}=\exp \left(-\mathrm{i} \beta_{j} d\right)\left(\beta_{j}\right.$ being the wave number, $d$ being the length of the substructure). Notice that the wave shapes $\left\{\phi_{j}\right\}_{j}$ represent vectors of kinematic/mechanical quantities expressed on the substructure boundaries only, i.e., they do not describe the motion at the internal nodes of the substructures.

The parameters $\left\{\left(\mu_{j}, \phi_{j}\right)\right\}_{j}$ are referred to as the wave modes of a periodic structure. In fact, there are twice as many wave modes as the number of DOFs contained on the left or right boundary of a substructure, i.e., $2 n$ wave modes. 
Finally note that the wave modes depend on the frequency, which is explained since the matrix $\mathbf{S}$ depends itself on the frequency.

\subsubsection{Computation of wave modes}

As pointed out in $[23,13]$, a direct computation of the eigenvalues and eigenvectors of the matrix $\mathbf{S}$ can be prone to numerical problems, which is explained because the matrix of eigenvectors is usually ill-conditioned. The issue lies in the fact that the eigenvectors are expressed in terms of displacement/rotation and force/moment components, whose values can be largely disparate. To circumvent this, a better conditioned generalized eigenproblem is usually considered as [23]:

$\mathbf{N w}_{j}=\mu_{j} \mathbf{L} \mathbf{w}_{j} \quad, \quad \operatorname{det}\left(\mathbf{N}-\mu_{j} \mathbf{L}\right)=0$,

where

$\mathbf{L}=\left[\begin{array}{cc}\mathbf{I}_{n} & \mathbf{0} \\ -\mathbf{D}_{\mathrm{LL}}^{*} & -\mathbf{D}_{\mathrm{LR}}^{*}\end{array}\right] \quad, \quad \mathbf{N}=\left[\begin{array}{cc}\mathbf{0} & \mathbf{I}_{n} \\ \mathbf{D}_{\mathrm{RL}}^{*} & \mathbf{D}_{\mathrm{RR}}^{*}\end{array}\right]$.

In Eq. (8) the eigenvectors $\left\{\mathbf{w}_{j}\right\}_{j}$ relate the displacements/rotations of the substructure, only. The determination of the eigenvectors of the matrix $\mathbf{S}$ simply follows as

$\phi_{j}=\mathbf{L w}_{j}$.

It is worth recalling that the wave modes depend on the frequency, i.e., they need to be computed for each discrete frequency involved within the frequency bands of interest. Also, the condensed dynamic stiffness matrix $\mathbf{D}^{*}$ of a substructure (Eq. (4)) is to be evaluated for each discrete frequency, prior to the computation of wave modes. The related CPU times may be cumbersome, considering that the number of internal DOFs of the substructure and the number of discrete frequencies analyzed are usually large. To circumvent this issue, the Craig-Bampton (CB) method can be used [3]. The procedure consists in making use of the static modes of the substructure and a reduced number of fixed interface modes. As a result, the computation of $\mathbf{D}^{*}$ can be sped up in a drastic way. The selection of the fixed interface modes is achieved by considering those whose eigenfrequencies are below a certain frequency threshold. From the practical point of view, the number of fixed interface modes to be retained can be roughly estimated by performing a convergence analysis of the WFE method for expressing the FRF of a periodic structure in the vicinity of the maximum frequency of the frequency band of interest.

\subsubsection{Properties of wave modes and conventions}

Due to the fact that the matrix $\mathbf{S}$ is symplectic, its eigenvalues come in pairs as $\mu_{j}$ and $1 / \mu_{j}$. Besides, since the substructures are damped, the magnitudes of the eigenvalues are different from unity. Thus, the eigenvalues can be partitioned as $\left\{\mu_{j}\right\}_{j=1, \ldots, n} \cup\left\{\mu_{j}^{\star}\right\}_{j=1, \ldots, n}$, where $\left|\mu_{j}\right|<1$ and $\mu_{j}^{\star}=1 / \mu_{j}$. In matrix notation, this is written as

$\mu^{\star}=\mu^{-1}$,

where $\boldsymbol{\mu}=\operatorname{diag}\left\{\mu_{j}\right\}_{j=1, \ldots, n}, \boldsymbol{\mu}^{\star}=\operatorname{diag}\left\{\mu_{j}^{\star}\right\}_{j=1, \ldots, n}$ and $\|\boldsymbol{\mu}\|_{2}<1$. Also, the wave shapes associated to the eigenvalues $\left\{\mu_{j}\right\}_{j}$ and $\left\{\mu_{j}^{\star}\right\}_{j}$ are denoted as $\left\{\boldsymbol{\phi}_{j}\right\}_{j}$ and $\left\{\boldsymbol{\phi}_{j}^{\star}\right\}_{j}$. In fact, the wave modes $\left\{\left(\mu_{j}, \phi_{j}\right)\right\}_{j=1, \ldots, n}$ may be understood as those traveling towards the right direction of a periodic structure, the wave modes $\left\{\left(\mu_{j}^{\star}, \phi_{j}^{\star}\right)\right\}_{j=1, \ldots, n}$ being those traveling in the opposite sense (Fig. 1).

In matrix notations, the matrices of wave shapes are denoted as $\boldsymbol{\Phi}=\left[\phi_{1} \cdots \phi_{n}\right]$ and $\boldsymbol{\Phi}^{\star}=\left[\phi_{1}^{\star} \cdots \phi_{n}^{\star}\right]$, which can be partitioned as

$\boldsymbol{\Phi}=\left[\begin{array}{l}\boldsymbol{\Phi}_{\mathrm{q}} \\ \boldsymbol{\Phi}_{\mathrm{F}}\end{array}\right] \quad, \quad \boldsymbol{\Phi}^{\star}=\left[\begin{array}{l}\boldsymbol{\Phi}_{\mathrm{q}}^{\star} \\ \boldsymbol{\Phi}_{\mathrm{F}}^{\star}\end{array}\right]$,

where the subscripts $q$ and $\mathrm{F}$ refer to the displacement/rotation and force/moment components, respectively. Also, $\boldsymbol{\Phi}_{\mathrm{q}}, \boldsymbol{\Phi}_{\mathrm{q}}^{\star}$, $\boldsymbol{\Phi}_{\mathrm{F}}$ and $\boldsymbol{\Phi}_{\mathrm{F}}^{\star}$ are square $n \times n$ matrices, which are full rank [13].

Finally note that each pair of wave shapes $\left(\phi_{j}, \phi_{j}^{\star}\right)$ can be normalized with respect to the symplectic scalar product $\phi_{j}^{\star T} \mathbf{J} \phi_{j}$, which is achieved in this way [23]:

$\phi_{j} \rightarrow \frac{\phi_{j}}{\left(\phi_{j}^{\star T} \mathbf{J} \phi_{j}\right)^{1 / 2}} \quad, \quad \phi_{j}^{\star} \rightarrow \frac{\phi_{j}^{\star}}{\left(\phi_{j}^{\star T} \mathbf{J} \phi_{j}\right)^{1 / 2}}$.

\subsubsection{Case of symmetric substructures}

Consider a periodic structure composed of substructures that are symmetric with respect to their mid-plane (Fig. 2). In that case, an exact analytical relation can be derived which links the wave shapes traveling in right and left directions as

$\Phi_{\mathrm{q}}^{\star}=\mathcal{R} \Phi_{\mathrm{q}} \quad, \quad \boldsymbol{\Phi}_{\mathrm{F}}^{\star}=-\mathcal{R} \boldsymbol{\Phi}_{\mathrm{F}}$.

Here, $\mathcal{R}$ is a $n \times n$ diagonal symmetry transformation matrix (with 1 or -1 as components), which is such that $\mathcal{R} \mathcal{R}=\mathbf{I}$. A proof of Eq. (14) follows from the fact that the block components of the condensed dynamic stiffness matrix $\mathbf{D}^{*}$ (Eq. (4)) are linked as $\mathbf{D}_{\mathrm{RR}}^{*}=\mathcal{R}^{T} \mathbf{D}_{\mathrm{LL}}^{*} \mathcal{R}$ and $\mathbf{D}_{\mathrm{RL}}^{*}=\mathcal{R}^{T} \mathbf{D}_{\mathrm{LR}}^{*} \mathcal{R}$. However, there's no need to assume that the FE meshes of the substructures are symmetric as long as the mesh density, inside the substructures, is high enough ${ }^{1}$. The issue can be viewed as ensuring that the FE meshes are able to accurately capture the shapes of the fixed interface modes involved for describing $\mathbf{D}^{*}$ (see comments after Eq. (10)).

1 In other words, it is expected that the condensed dynamic stiffness matrix issued from an arbitrary mesh is almost the same as the one involved by a symmetric mesh, provided that the number of internal nodes is large enough. 
The feature of Eq. (14), along with the consideration of Eq. (11), is that it strictly enforces the coherence between the right-going and left-going wave modes. This means that each eigenvector $\boldsymbol{\Phi}_{j}^{\star}$ is to be evaluated by considering Eq. (14), rather than considering the solution of the eigenproblem (8). Also, each eigenvalue $\mu_{j}^{\star}$ is to be evaluated by means of Eq. (11), i.e. $\mu_{j}^{\star}=1 / \mu_{j}$. It will be shown in Section 4 that the non-consideration of the analytic relations (14) and (11) is source of numerical ill-conditioning, which appears dramatic for describing the forced response of structures.

\subsection{Forced response computation}

\subsubsection{Wave mode expansion}

Consider a periodic structure composed of $N$ substructures, and denote as $k(k=1, \ldots, N+1)$ a substructure boundary, i.e., either a coupling interface between two substructures, or one end of the periodic structure. The key idea here is to expand the vectors of displacements/rotations $\mathbf{q}^{(k)}$ and forces/moments $\mathbf{F}^{(k)}$, on the substructure boundary $k$, in the wave basis $\left\{\phi_{j}\right\}_{j} \cup\left\{\phi_{j}^{\star}\right\}_{j}$. In matrix form, this wave expansion is expressed as

$$
\begin{aligned}
& \mathbf{q}^{(k)}=\boldsymbol{\Phi}_{\mathbf{q}} \mathbf{Q}^{(k)}+\boldsymbol{\Phi}_{\mathbf{q}}^{\star} \mathbf{Q}^{\star(k)}, \\
& \pm \mathbf{F}^{(k)}=\boldsymbol{\Phi}_{\mathbf{F}} \mathbf{Q}^{(k)}+\boldsymbol{\Phi}_{\mathbf{F}}^{\star} \mathbf{Q}^{\star(k)} .
\end{aligned}
$$

In Eq. (16), the sign ahead of $\mathbf{F}$ results from the choice of description considered - i.e., either the right or left boundaries of the substructures (cf. Eq. (2)). In Eqs. (15) and (16), the terms $\mathbf{Q}^{(k)}$ and $\mathbf{Q}^{\star(k)}$ are vectors of wave amplitudes. By considering the properties mentioned after Eq. (7), it turns out that

$\mathbf{Q}^{(k)}=\boldsymbol{\mu}^{k-1} \mathbf{Q} \quad, \quad \mathbf{Q}^{\star(k)}=\boldsymbol{\mu}^{N+1-k} \mathbf{Q}^{\star}$,

where

$$
\mathbf{Q}=\mathbf{Q}^{(1)}, \quad \mathbf{Q}^{\star}=\mathbf{Q}^{\star(N+1)}
$$

Here, $\mathbf{Q}$ and $\mathbf{Q}^{\star}$ represent the vectors of wave amplitudes at the left and right ends of the whole structure, respectively. The convention provided by Eq. (18) is introduced as a means to simplify the subsequent derivations made in the paper. Thus, Eqs. (15) and (16) can be rewritten as

$$
\begin{aligned}
& \mathbf{q}^{(k)}=\boldsymbol{\Phi}_{\mathrm{q}} \boldsymbol{\mu}^{k-1} \mathbf{Q}+\boldsymbol{\Phi}_{\mathrm{q}}^{\star} \boldsymbol{\mu}^{N+1-k} \mathbf{Q}^{\star}, \\
& \pm \mathbf{F}^{(k)}=\boldsymbol{\Phi}_{\mathrm{F}} \boldsymbol{\mu}^{k-1} \mathbf{Q}+\boldsymbol{\Phi}_{\mathrm{F}}^{\star} \boldsymbol{\mu}^{N+1-k} \mathbf{Q}^{\star} .
\end{aligned}
$$

\subsubsection{Wave-based matrix formulation}

The key idea behind the WFE strategy is to compute the vectors of wave amplitudes $\mathbf{Q}$ and $\mathbf{Q}^{\star}$, which ultimately provide, from Eqs. (19) and (20), the vectors $\mathbf{q}^{(k)}$ and $\mathbf{F}^{(k)}$ on any substructure boundary $k$. If needed, the vector of displacements/rotations at the internal nodes of a substructure can be obtained from those defined on its boundaries ${ }^{2}$. The computation of the vectors $\mathbf{Q}$ and $\mathbf{Q}^{\star}$ follows from the consideration of the boundary conditions of the structure. The procedure has been proposed in [13] when Neumann and Dirichlet conditions are dealt with. The same strategy holds for arbitrary boundary conditions, e.g., surface impedances [14]. Such boundary conditions can be formulated as

$\mathbf{Y} \mathbf{q}_{\mathrm{L}}^{(1)}+\mathbf{Z F}_{\mathrm{L}}^{(1)}=\mathbf{Y}_{0} \mathbf{q}_{0}+\mathbf{Z}_{0} \mathbf{F}_{0}$

$\mathbf{Y}^{\star} \mathbf{q}_{\mathrm{R}}^{(N+1)}+\mathbf{Z}^{\star} \mathbf{F}_{\mathrm{R}}^{(N+1)}=\mathbf{Y}_{0}^{\star} \mathbf{q}_{0}^{\star}+\mathbf{Z}_{0}^{\star} \mathbf{F}_{0}^{\star}$,

where $\mathbf{Y}, \mathbf{Y}^{\star}, \mathbf{Y}_{0}, \mathbf{Y}_{0}^{\star}, \mathbf{Z}, \mathbf{Z}^{\star}, \mathbf{Z}_{0}, \mathbf{Z}_{0}^{\star}$ are $n \times n$ matrices whose meanings actually depend on the kind of application studied. For the sake of clarity, the matrices $\mathbf{Y}, \mathbf{Y}_{0}, \mathbf{Z}$ and $\mathbf{Z}_{0}$ are explicitly expressed hereafter for some simple cases (expressions of $\mathbf{Y}^{\star}, \mathbf{Y}_{0}^{\star}, \mathbf{Z}^{\star}$ and $\mathbf{Z}_{0}^{\star}$ can be simply deduced from this analysis):

- Prescribed vector of displacements/rotations $\mathbf{q}_{0}$ :

$$
\mathbf{q}_{\mathrm{L}}^{(1)}=\mathbf{q}_{0} \Rightarrow \mathbf{Y}=\mathbf{I}, \mathbf{Y}_{0}=\mathbf{I}, \mathbf{Z}=\mathbf{0}, \mathbf{Z}_{0}=\mathbf{0} .
$$

- Prescribed vector of forces/moments $\mathbf{F}_{0}$ :

$$
\mathbf{F}_{\mathrm{L}}^{(1)}=\mathbf{F}_{0} \quad \Rightarrow \quad \mathbf{Y}=\mathbf{0}, \mathbf{Y}_{0}=\mathbf{0}, \mathbf{Z}=\mathbf{I}, \mathbf{Z}_{0}=\mathbf{I}
$$

- Matrix of surface impedances $\mathcal{Z}$ :

$$
\begin{aligned}
& \mathbf{F}_{\mathrm{L}}^{(1)}=-i \omega \mathcal{Z}\left(\mathbf{q}_{\mathrm{L}}^{(1)}-\mathbf{q}_{0}\right) \\
& \Rightarrow \quad \mathbf{Y}=i \omega \mathcal{Z}, \mathbf{Y}_{0}=i \omega \mathcal{Z}, \mathbf{Z}=\mathbf{I}, \mathbf{Z}_{0}=\mathbf{0} .
\end{aligned}
$$

Introducing the wave expansions (19) and (20) into Eqs. (21) and (22) provides, after some algebra, the following wavebased matrix equation:

$$
\left[\begin{array}{cc}
\mathbf{I} & \mathbf{X} \boldsymbol{\mu}^{N} \\
\mathbf{X}^{\star} \boldsymbol{\mu}^{N} & \mathbf{I}
\end{array}\right]\left[\begin{array}{c}
\mathbf{Q} \\
\mathbf{Q}^{\star}
\end{array}\right]=\left[\begin{array}{c}
\mathbf{G} \\
\mathbf{G}^{\star}
\end{array}\right]
$$

where

$$
\begin{aligned}
& \mathbf{X}=\left(\mathbf{Y} \boldsymbol{\Phi}_{\mathrm{q}}-\mathbf{Z} \boldsymbol{\Phi}_{\mathrm{F}}\right)^{-1}\left(\mathbf{Y} \boldsymbol{\Phi}_{\mathrm{q}}^{\star}-\mathbf{Z} \boldsymbol{\Phi}_{\mathrm{F}}^{\star}\right), \\
& \mathbf{X}^{\star}=\left(\mathbf{Y}^{\star} \boldsymbol{\Phi}_{\mathrm{q}}^{\star}+\mathbf{Z}^{\star} \boldsymbol{\Phi}_{\mathrm{F}}^{\star}\right)^{-1}\left(\mathbf{Y}^{\star} \boldsymbol{\Phi}_{\mathrm{q}}+\mathbf{Z}^{\star} \boldsymbol{\Phi}_{\mathrm{F}}\right),
\end{aligned}
$$

2 This is achieved by considering the components $\mathbf{D}_{\text {IB }}$ and $\mathbf{D}_{\text {II }}$ of the dynamic stiffness matrix of the substructure, i.e, $\mathbf{q}_{\mathrm{I}}=$ $-\mathbf{D}_{\mathrm{II}}^{-1} \mathbf{D}_{\mathrm{IB}} \mathbf{q}_{\mathrm{B}}$. Alternatively, the Craig-Bampton procedure may be used to circumvent the direct computation of the matrix inverse $\mathbf{D}_{\text {II }}^{-1}$ [3]. 


$$
\begin{aligned}
& \mathbf{G}=\left(\mathbf{Y} \boldsymbol{\Phi}_{\mathrm{q}}-\mathbf{Z} \boldsymbol{\Phi}_{\mathrm{F}}\right)^{-1}\left(\mathbf{Y}_{0} \mathbf{q}_{0}+\mathbf{Z}_{0} \mathbf{F}_{0}\right), \\
& \mathbf{G}^{\star}=\left(\mathbf{Y}^{\star} \boldsymbol{\Phi}_{\mathrm{q}}^{\star}+\mathbf{Z}^{\star} \boldsymbol{\Phi}_{\mathrm{F}}^{\star}\right)^{-1}\left(\mathbf{Y}_{0}^{\star} \mathbf{q}_{0}^{\star}+\mathbf{Z}_{0}^{\star} \mathbf{F}_{0}^{\star}\right) .
\end{aligned}
$$

To derive Eq. (26), it has been assumed that the matrices $\left(\mathbf{Y} \boldsymbol{\Phi}_{\mathrm{q}}-\mathbf{Z} \boldsymbol{\Phi}_{\mathrm{F}}\right)$ and $\left(\mathbf{Y}^{\star} \boldsymbol{\Phi}_{\mathrm{q}}^{\star}+\mathbf{Z}^{\star} \boldsymbol{\Phi}_{\mathrm{F}}^{\star}\right)$ are invertible. This can be proved when the structure boundaries are subject to prescribed displacements/rotations or prescribed forces/moments (Eqs. (23) and (24)), because the matrices $\boldsymbol{\Phi}_{\mathrm{q}}, \boldsymbol{\Phi}_{\mathrm{F}}$, $\boldsymbol{\Phi}_{\mathrm{q}}^{\star}$ and $\boldsymbol{\Phi}_{\mathrm{F}}^{\star}$ are full rank [13]; the fact that $\left(\mathbf{Y} \boldsymbol{\Phi}_{\mathrm{q}}-\mathbf{Z} \boldsymbol{\Phi}_{\mathrm{F}}\right)$ and $\left(\mathbf{Y}^{\star} \boldsymbol{\Phi}_{\mathrm{q}}^{\star}+\mathbf{Z}^{\star} \boldsymbol{\Phi}_{\mathrm{F}}^{\star}\right)$ are invertible seems also to be verified in other cases, for instance when the structure boundaries are subject to surface impedances (Eq. (25)), except maybe on rare occasions (e.g., when $i \omega \mathcal{Z} \boldsymbol{\Phi}_{\mathrm{q}}-\boldsymbol{\Phi}_{\mathrm{F}}=\mathbf{0}$ ) which will not be considered here. The motivation behind the use of the matrix inverses $\left(\mathbf{Y} \boldsymbol{\Phi}_{\mathrm{q}}-\mathbf{Z} \boldsymbol{\Phi}_{\mathrm{F}}\right)^{-1}$ and $\left(\mathbf{Y}^{\star} \boldsymbol{\Phi}_{\mathrm{q}}^{\star}+\mathbf{Z}^{\star} \boldsymbol{\Phi}_{\mathrm{F}}^{\star}\right)^{-1}$ is to make the matrix occurring on the left hand side of Eq. (26) well conditioned (this interesting feature is emphasized in $[13,14])$.

In condensed form, the matrix system (26) is expressed as $\mathbf{A} \mathcal{Q}=\mathcal{F}$, where $\mathcal{Q}=\left[\mathbf{Q}^{T} \mathbf{Q}^{\star T}\right]^{T}$. The determination of the vectors of wave amplitudes $\mathbf{Q}$ and $\mathbf{Q}^{\star}$ follows as $\mathcal{Q}=\mathbf{A}^{-1} \mathcal{F}$. The consideration of several waveguides, coupled to each other directly, or by means of coupling elements, does not bring additional difficulties. This requires one to consider a matrix system quite similar (but with a larger size) to Eq. (26), i.e., of the form $\mathbf{A} \mathcal{Q}=\mathcal{F}$ where $\mathcal{Q}=\left[\mathbf{Q}_{1}^{T} \mathbf{Q}_{1}^{\star T} \mathbf{Q}_{2}^{T} \mathbf{Q}_{2}^{\star T} \cdots \mathbf{Q}_{M}^{T} \mathbf{Q}_{M}^{\star T}\right]^{T}\left(\mathbf{Q}_{i}\right.$ and $\mathbf{Q}_{i}^{\star}$ being the vectors of wave amplitudes for a given waveguide $i, M$ being the number of waveguides involved).

\subsubsection{Discussion}

The relevance of the wave-based matrix formulation (Eq. (26)) has been highlighted in past works $[13,14]$. It has proved to be accurate for computing the harmonic forced response of single and coupled straight structures over wide frequency bands. In those works, symmetric substructures were of concern, and the analytical relations (14) and (11) were considered. As explained in Section 2.1.4, the key idea behind the use of these relations is to strictly enforce the coherence between the right-going and left-going wave modes: this means that two dual wave modes are expected to have exactly the same velocities, while their shapes are expected to be symmetric to each other. Should these equations not be considered, numerical dispersion is likely to occur between these wave modes. The issue concerns the determination of the matrices $\mathbf{X}$ and $\mathbf{X}^{\star}$ (Eqs. (27) and (28)) - these can be viewed as the projections of some spaces spanned by the left-going wave shapes onto some spaces spanned by the right-going wave shapes, or vice versa - which become nearly rank deficient, hence impacting the numerical conditioning of the matrix system (26). This issue will be highlighted in Section 4.

\section{Interpolation strategy}

\subsection{Motivation and framework}

Within the WFE framework, the forced response computation of periodic structures requires one to express a matrix equation of the form $\mathbf{A} \mathcal{Q}=\mathcal{F}$ (Eq. (26)) and its solution $\mathcal{Q}=\mathbf{A}^{-1} \mathcal{F}$, for several discrete frequencies. In doing so, the following numerical tasks need to be undertaken:

(i) Computation of the condensed dynamic stiffness matrix $\mathrm{D}^{*}$ of a substructure (see comments after Eq. (10));

(ii) Computation of the eigenproblem (8);

(iii) Computation of the matrix $\mathbf{A}$ and its inverse.

The numerical tasks (i)-(iii) may be time consuming to describe the FRFs of structures over wide frequency bands, i.e., when these FRFs exhibit many resonance phenomena. This is especially true when periodic structures are dealt with, since the substructures exhibit complex local dynamics that are of primary importance, even at low frequencies. This means that the number of discrete frequencies involved to describe these FRFs accurately is usually large (e.g., more than one thousand).

To reduce the computational cost involved by the WFE method, a strategy is proposed to interpolate the vector of displacements/rotations $\mathbf{q}^{(k)}$ or the vector of forces/moments $\mathbf{F}^{(k)}$, on any substructure boundary $k$, at a reduced number of discrete frequencies - namely, the interpolation points. A linear interpolation scheme is used, between two consecutive interpolation points, to approximate these vectors at intermediate points where the numerical tasks (ii) and (iii) do not need to be undertaken. The motivation behind the use of a linear interpolation scheme is that it can be easily applied to the solutions issued from the wave-based matrix equation (26). The procedure is detailed hereafter.

The proposed strategy starts by considering a coarse set of discrete angular frequencies $\left\{\Omega_{p}\right\}_{p}$ as interpolation points, which are uniformly spread on a whole frequency band with a constant angular frequency step $\Delta \Omega$. The WFE solution - i.e., the vectors of displacements/rotations and forces/moments provided by Eqs. (19) and (20) - is explicitly computed at the interpolation points $\left\{\Omega_{p}\right\}_{p}$ by taking into account the numerical tasks (i)-(iii). Besides, the WFE solution is approximated at each intermediate point $\omega_{s}$ between two consecutive interpolation points $\Omega_{p}$ and $\Omega_{p+1}$, as follows:

$$
\tilde{\mathbf{q}}^{(k)}\left(\omega_{s}\right)=\mathbf{q}^{(k)}\left(\Omega_{p}\right)+\frac{\omega_{s}-\Omega_{p}}{\Delta \Omega_{p}}\left(\mathbf{q}^{(k)}\left(\Omega_{p+1}\right)-\mathbf{q}^{(k)}\left(\Omega_{p}\right)\right),
$$

where $\Delta \Omega_{p}=\Omega_{p+1}-\Omega_{p}$. Here, the vector of forces/moments has been omitted for the sake of conciseness. The strategy uses adaptive refinement, i.e., any intermediate point 
$\omega_{s}$ is considered as a new interpolation point when the relative error, between the interpolated solution $\tilde{\mathbf{q}}^{(k)}\left(\omega_{s}\right)$ and the exact one $\mathbf{q}^{(k)}\left(\omega_{s}\right)$, exceeds a certain tolerance threshold. For those new interpolation points, the WFE solution is explicitly computed by means of the numerical tasks (i)(iii). Thus, a new set of interpolation points $\left\{\Omega_{p}\right\}_{p}$ is defined and the approximated solution is re-expressed (Eq. (31)). The key challenge here is to provide a good indicator of the relative error between the interpolated solution $\tilde{\mathbf{q}}^{(k)}\left(\omega_{s}\right)-$ i.e, which results from the initial coarse set of interpolation points - and the exact solution $\mathbf{q}^{(k)}\left(\omega_{s}\right)$, given that the latter is not explicitly known. This error indicator should be expressed as

$\epsilon_{s} \approx \frac{\left\|\tilde{\mathbf{q}}^{(k)}\left(\omega_{s}\right)-\mathbf{q}^{(k)}\left(\omega_{s}\right)\right\|}{\left\|\mathbf{q}^{(k)}\left(\omega_{s}\right)\right\|} \quad \forall k$.

In order to make the procedure efficient, the CPU time involved in the evaluation of the error indicator $\epsilon_{s}$, for each discrete angular frequency $\omega_{s}$, should be small compared to the computation of the direct solution $\mathbf{q}^{(k)}\left(\omega_{s}\right)$. Also, the expression of $\epsilon_{s}$ should be independent of the substructure boundary number $k$. This issue is addressed in Section 3.2. It is shown that a simple expression of $\epsilon_{s}$ can be obtained. The formulation of this error indicator uses the fact that periodic structures are dealt with.

\subsection{Error indicator}

The question of how good is the interpolated solution $\tilde{\mathbf{q}}^{(k)}\left(\omega_{s}\right)$, compared to the exact one $\mathbf{q}^{(k)}\left(\omega_{s}\right)$, is addressed in the following way.

Consider a periodic structure and two substructure boundaries $k$ and $k+1$, with the related vectors of displacements/rotations approximated as $\tilde{\mathbf{q}}^{(k)}\left(\omega_{s}\right)$ and $\tilde{\mathbf{q}}^{(k+1)}\left(\omega_{s}\right)$ (Eq. (31)). The vector of forces/moments on the substructure boundary $k(k=1, \ldots, N)$, which results from these vectors of displacements/rotations, is given by

$\hat{\mathbf{F}}^{(k)}\left(\omega_{s}\right)=\mathbf{D}_{\mathrm{LL}}^{*}\left(\omega_{s}\right) \tilde{\mathbf{q}}^{(k)}\left(\omega_{s}\right)+\mathbf{D}_{\mathrm{LR}}^{*}\left(\omega_{s}\right) \tilde{\mathbf{q}}^{(k+1)}\left(\omega_{s}\right)$,

where $\mathbf{D}_{\mathrm{LL}}^{*}$ and $\mathbf{D}_{\mathrm{LR}}^{*}$ are block components of the condensed dynamic stiffness matrix of a substructure, Eq. (4). In contrast, the interpolated vector of forces/moments is given by

$\tilde{\mathbf{F}}^{(k)}\left(\omega_{s}\right)=\mathbf{F}^{(k)}\left(\Omega_{p}\right)+\frac{\omega_{s}-\Omega_{p}}{\Delta \Omega_{p}}\left(\mathbf{F}^{(k)}\left(\Omega_{p+1}\right)-\mathbf{F}^{(k)}\left(\Omega_{p}\right)\right)$.

The present error analysis is based on the idea to compare $\hat{\mathbf{F}}^{(k)}\left(\omega_{s}\right)$ with $\tilde{\mathbf{F}}^{(k)}\left(\omega_{s}\right)$. A large relative error between these two quantities would mean that the interpolated solution is not accurate. In other words, the relative error made at the level of one substructure is expected to be

$\frac{\left\|\tilde{\mathbf{F}}^{(k)}\left(\omega_{s}\right)-\hat{\mathbf{F}}^{(k)}\left(\omega_{s}\right)\right\|}{\left\|\hat{\mathbf{F}}^{(k)}\left(\omega_{s}\right)\right\|}$.

A more accurate indicator is proposed here which takes into account the displacement/rotation part of the WFE solution. This is achieved by weighting the force/moment components $\tilde{\mathbf{F}}^{(k)}\left(\omega_{s}\right)$ and $\hat{\mathbf{F}}^{(k)}\left(\omega_{s}\right)$ by the displacement/rotation component $\tilde{\mathbf{q}}^{(k)}\left(\omega_{s}\right)$. This leads to the following relative error:

$\frac{\left|\tilde{\mathbf{q}}^{(k)}\left(\omega_{s}\right)^{H} \tilde{\mathbf{F}}^{(k)}\left(\omega_{s}\right)-\tilde{\mathbf{q}}^{(k)}\left(\omega_{s}\right)^{H} \hat{\mathbf{F}}^{(k)}\left(\omega_{s}\right)\right|}{\left|\tilde{\mathbf{q}}^{(k)}\left(\omega_{s}\right)^{H} \hat{\mathbf{F}}^{(k)}\left(\omega_{s}\right)\right|}$,

where $\tilde{\mathbf{q}}^{(k)}\left(\omega_{s}\right)^{H}$ is the conjugate transpose of $\tilde{\mathbf{q}}^{(k)}\left(\omega_{s}\right)$. Notice that the relative error (36) is to be considered for each substructure boundary $k$ involved in a whole periodic structure. Regarding the whole structure, it is then proposed to assess the error indicator $\epsilon_{s}$ as

$\epsilon_{s}=N \times \max _{k}\left\{\frac{\left|\tilde{\mathbf{q}}^{(k)}\left(\omega_{s}\right)^{H} \tilde{\mathbf{F}}^{(k)}\left(\omega_{s}\right)-\tilde{\mathbf{q}}^{(k)}\left(\omega_{s}\right)^{H} \hat{\mathbf{F}}^{(k)}\left(\omega_{s}\right)\right|}{\left|\tilde{\mathbf{q}}^{(k)}\left(\omega_{s}\right)^{H} \hat{\mathbf{F}}^{(k)}\left(\omega_{s}\right)\right|}\right\}$,

where $N$ is the number of substructures considered. To formulate Eq. (37), the maximum of the relative errors, among all the substructures, has been considered and multiplied by the number of substructures.

The CPU time involved to calculate the error indicator $\epsilon_{s}$ is mainly related to the computation of the condensed dynamic stiffness matrix $\mathbf{D}^{*}$ of a substructure, i.e., the numerical tasks (ii) and (iii) mentioned at the beginning of Section 3.1 are not considered. The relevance of the proposed error indicator $\epsilon_{s}$ will be highlighted in Section 4.

\subsection{Implementation}

As explained previously, the proposed approach consists in evaluating first the solution of the WFE method at a reduced number of discrete angular frequencies $\left\{\Omega_{p}\right\}_{p}$ that are equally spaced by means of a constant angular frequency step $\Delta \Omega_{p}=\Delta \Omega$. The WFE solution is then approximated at each intermediate point $\omega_{s}$, between two consecutive interpolation points $\Omega_{p}$ and $\Omega_{p+1}$ (Eq. (31)). An error indicator $\epsilon_{s}$ is considered to assess the accuracy of the interpolated solution at the intermediate points (Eq. (37)). The strategy uses adaptive refinement in the sense that any intermediate point $\omega_{s}$ is considered as a new interpolation point when the error indicator $\epsilon_{s}$ exceeds a certain tolerance threshold $\mathcal{E}$. Then a new set of interpolation points $\left\{\Omega_{p}\right\}_{p}$ is defined and a new linear interpolation is considered (Eq. (31)). At this stage, it is assumed that the error indicator $\epsilon_{s}$ remains 
smaller than the tolerance threshold $\mathcal{E}$ at the new intermediate points. The relevance of this assumption will be highlighted in Section 4. The algorithm procedure of the proposed interpolation strategy is summarized as follows:

1. Set the value of the initial angular frequency step $\Delta \Omega_{p}=$ $\Delta \Omega$ and the value of the tolerance threshold $\mathcal{E}$;

2. Compute explicitly the WFE solution $\mathbf{q}^{(k)}$ for each interpolation point $\Omega_{p}$ : numerical tasks (i-iii) (Section 3.1), Eq. (19);

3. Evaluate the approximated solution $\tilde{\mathbf{q}}^{(k)}$ for each intermediate point $\left.\omega_{s} \in\right] \Omega_{p}, \Omega_{p+1}[$ : Eq. (31);

4. Compute the error indicator $\epsilon_{s}$ for each intermediate point $\omega_{s}$ : numerical task (i) (Section 3.1), Eq. (37);

5. Compute explicitly the WFE solution $\mathbf{q}^{(k)}$ for each intermediate point $\omega_{s}$ where $\epsilon_{s}>\mathcal{E}$, and set $\omega_{s}$ as a new interpolation point $\left(\omega_{s} \rightarrow\left\{\Omega_{p}\right\}_{p}\right)$ : numerical tasks (iiii) (Section 3.1), Eq. (19);

6. Interpolate the WFE solution by considering the new interpolation points $\left\{\Omega_{p}\right\}_{p}$ and the new angular frequency steps $\left\{\Delta \Omega_{p}\right\}_{p}$ : Eq. (31).

\section{Numerical experiments}

\subsection{Introduction}

The WFE strategies proposed in Sections 2 and 3 are applied to assess the dynamic behavior of the following periodic structures: (i) a beam with holes (Fig. 3); (ii) a curved stiffened panel (Fig. 8). The FE meshes of the related substructures, as displayed in Fig. 4 and Fig. 9, contain many internal DOFs. Here, the COMSOL Multiphysics ${ }^{\circledR}$ software is used to express the mass and stiffness matrices of each substructure. Those matrices are then post-treated using MATLAB ${ }^{\circledR}$ with a view to computing the frequency forced response of the structures. Among the numerical tasks, which are carried out using MATLAB ${ }^{\circledR}$, is the computation of the condensed dynamic stiffness matrix $\mathbf{D}^{*}$ by means of the $\mathrm{CB}$ method, as discussed in Section 2.1.2.

The dynamic behavior of the periodic structures is investigated over wide frequency bands where global as well as local resonances, inside the substructures, are likely to occur. In other words, the FRFs are expected to be complex, i.e., with a large number of resonance and anti-resonance peaks that are not necessarily uniformly spread on the whole frequency bands. Notice that these FRFs are not known a priori. A modal analysis may however give a coarse indication about the number of resonance frequencies involved. The need to describe these FRFs accurately, especially around the resonance and anti-resonance peaks which can be sharp, requires one to discretize the frequency bands with a sufficient number of discrete frequencies.

For each test case, the norm of the velocity vector, on a given substructure boundary $k$, is computed by means of the WFE method using MATLAB ${ }^{\circledR}$. The norm of the velocity vector is defined as $\left\|\mathbf{i} \omega \mathcal{L} \mathbf{q}^{(k)}\right\|$, where $\mathcal{L}$ is a Boolean matrix that selects the displacement DOFs of the substructure boundary (i.e., not the rotation DOFs). The WFE solution is compared with the result of the FE method, also obtained using MATLAB ${ }^{\circledR}$, when the whole periodic structure is considered. Within the FE framework, the CB method is also used to calculate the condensed dynamic stiffness matrix $\mathbf{D}^{*}$ of each substructure. The FE model of the whole periodic structure hence follows from classic assembly procedures of the CB-based substructure models. In other words, both WFE and FE modelings make use of the same condensation procedure to compute $\mathbf{D}^{*}$. The comparison between the WFE and FE solutions is achieved by assessing the following relative error:

$\frac{\left\|i \omega \mathcal{L} \mathbf{q}^{(k)}-i \omega \mathcal{L} \mathbf{q}_{\mathrm{FE}}^{(k)}\right\|}{\left\|i \omega \mathcal{L} \mathbf{q}_{\mathrm{FE}}^{(k)}\right\|}$,

where $\mathcal{L} \mathbf{q}_{\mathrm{FE}}^{(k)}$ represents the displacement vector issued from the FE method.

Besides, the WFE-based interpolation strategy is considered (Section 3). Its efficiency, in terms of CPU time savings, is highlighted. Also, the accuracy of the error indicator $\epsilon_{s}$ (Eq. (37)) is highlighted in comparison with the true relative error, expressed as

$\frac{\left\|i \omega \mathcal{L} \tilde{\mathbf{q}}^{(k)}-i \omega \mathcal{L} \mathbf{q}_{\mathrm{FE}}^{(k)}\right\|}{\left\|i \omega \mathcal{L} \mathbf{q}_{\mathrm{FE}}^{(k)}\right\|}$.

\subsection{Beam with holes}

\subsubsection{Problem description}

The dynamic behavior of a beam structure, with a periodic inclusion of holes, is analyzed. The periodic structure is displayed in Fig. 3; it exhibits the following characteristics: length $1.5 \mathrm{~m}$, height $0.2 \mathrm{~m}$, thickness $2 \times 10^{-3} \mathrm{~m}$; density $7800 \mathrm{~kg} / \mathrm{m}^{3}$, Young's modulus $210 \times 10^{9} \mathrm{~Pa}$, Poisson's ratio 0.3 , loss factor $\eta=5 \times 10^{-3}$. The periodic structure is composed of $N=15$ similar substructures (Fig. 4), each of these containing two vertically placed holes of diameter $0.07 \mathrm{~m}$. The beam is clamped over its right end, and subject to an horizontal point force acting at the bottom side of the left end (Fig. 3).

Each substructure is meshed using 2D plane stress triangular elements of arbitrary size, with three nodes and two translational DOFs per node (Fig. 4). The substructure is discretized by means of $n=82$ DOFs for each left/right 


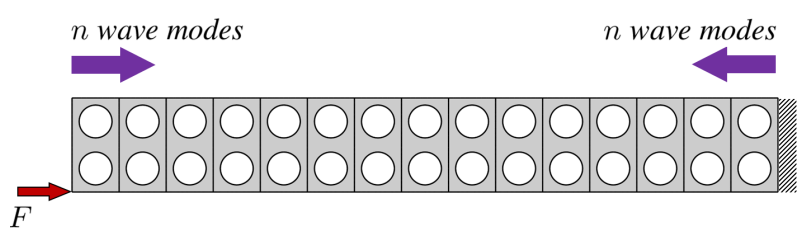

Fig. 3 Beam with holes.

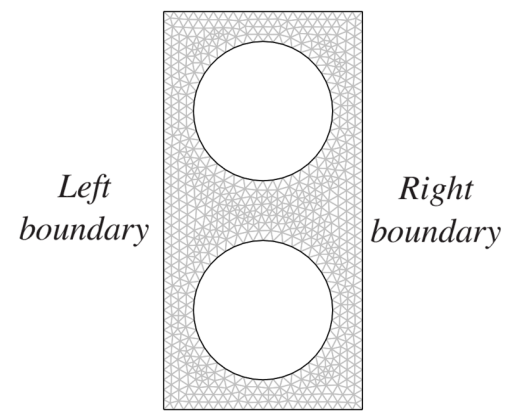

Fig. 4 FE model of a substructure (beam with holes).

boundary, and 1484 internal DOFs. The dynamic behavior of the periodic structure is analyzed over the frequency band $[1 \mathrm{~Hz}, 8000 \mathrm{~Hz}]$ by considering 8000 discrete frequencies that are equally spaced with a step of $1 \mathrm{~Hz}$. Here, the condensed dynamic stiffness matrix $\mathbf{D}^{*}$ of each substructure is expressed by considering the CB method with 50 fixed interface modes (Section 2.1.2). Hereafter, the conventional WFE method and the WFE-based interpolation strategy are applied to evaluate the norm of the velocity vector on the left end of the structure, i.e., $\left\|\mathbf{i} \omega \mathbf{q}^{(1)}\right\|$.

\subsubsection{WFE solution}

The WFE solution is obtained by solving the wave-based matrix equation (26) for each discrete frequency, and considering the wave expansion (19). Within the present framework, the wave-based matrix equation is expressed as

$\left[\begin{array}{cc}\mathbf{I} & \boldsymbol{\Phi}_{\mathrm{F}}^{-1} \boldsymbol{\Phi}_{\mathrm{F}}^{\star} \boldsymbol{\mu}^{N} \\ \boldsymbol{\Phi}_{\mathrm{q}}^{\star} \boldsymbol{\Phi}_{\mathrm{q}} \boldsymbol{\mu}^{N} & \mathbf{I}\end{array}\right]\left[\begin{array}{c}\mathbf{Q} \\ \mathbf{Q}^{\star}\end{array}\right]=\left[\begin{array}{c}-\mathbf{F}_{0} \\ \mathbf{0}\end{array}\right]$,

where $\mathbf{F}_{0}$ relates the vector of prescribed forces applied to the left end of the structure. The expression of the wavebased matrix equation results from Eqs. (27)-(30). The FRFs issued from the WFE and FE methods are displayed in Fig. 5. Also, the relative error between the WFE and FE solutions (Eq. (38)) is shown. For the sake of clarity, the maximum values of the relative error, over small frequency bands of length $100 \mathrm{~Hz}$, have been plotted. As shown, the WFE solution perfectly matches the reference FE solution over the whole frequency band where the relative error remains below $0.5 \%$. In terms of CPU times, it takes $868 s$ to describe the FRF of the periodic structure with the WFE method, against $2065 s$ with the FE method. These CPU times are obtained using MATLAB ${ }^{\circledR}$ with an Intel $^{\circledR}$ Core $^{\text {TM }}$ i7-3720QM processor. This means a reduction of $58 \%$ in benefit of the WFE method. These results give credit to the WFE method for computing the forced response of periodic structures.
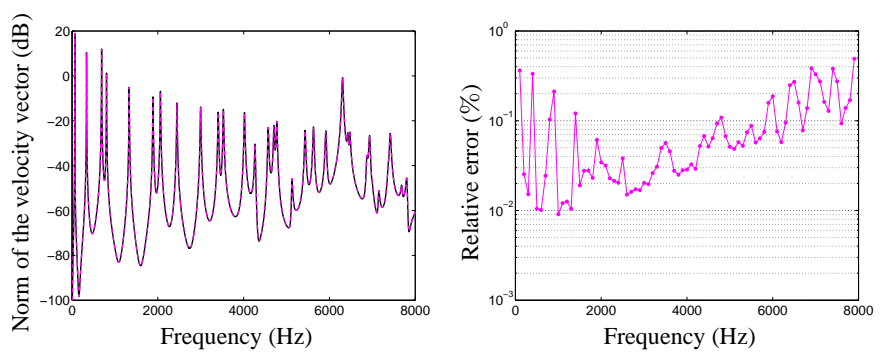

Fig. 5 FRF of the beam with holes: (Left figure) FE solution (-) and WFE solution (- - ); (Right figure) relative error of the WFE solution.

As pointed out in Sections 2.1.4 and 2.2.3, the non-consideration of the analytical relations (14) and (11) can be source of numerical problems. This issue is clearly highlighted in Fig. 6 when the FRF issued from the WFE method, as well as the condition number $\kappa(\mathbf{A})$ of the matrix $\mathbf{A}$ occurring on the left hand side of Eq. (40), are plotted. As seen, the WFE solution faces strong numerical instabilities, which is explained because the condition number appears tremendous compared to the regularized formulation, i.e., when Eqs. (14) and (11) are taken into account.
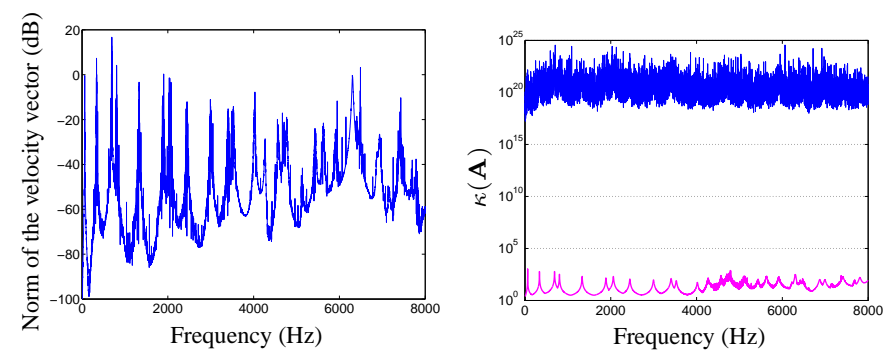

Fig. 6 FRF of the beam with holes: (Left figure) WFE solution without considering Eqs. (14) and (11); (Right figure) condition number of $\mathbf{A}$, without considering Eqs. (14) and (11) (- ) and by considering Eqs. (14) and (11) (־).

\subsubsection{WFE-based interpolated solution}

The interpolation strategy proposed in Section 3 is applied to describe the FRF of the periodic structure. In the present framework, a coarse set of discrete angular frequencies $\left\{\Omega_{p}\right\}_{p}$ is chosen so that the angular frequency step $\Delta \Omega$ is ten times larger than the one used in the previous simulations (i.e., 
$\Delta \Omega=10 H z)$. Intermediate points are considered which are equally spaced, with a step of $1 \mathrm{~Hz}$, between two consecutive interpolation points $\Omega_{p}$ and $\Omega_{p+1}$. Adaptive refinement is carried out (Section 3.3) by considering the error indicator $\epsilon_{s}$ (Eq. (37)) and a tolerance threshold $\mathcal{E}=10 \%$. The WFEbased interpolated solution is displayed in Fig. 7 along with the true relative error (Eq. (39)). It is seen that the WFEbased interpolated solution perfectly matches the FE solution. As expected, the true relative error appears bounded by the tolerance threshold $\mathcal{E}=10 \%$, except for two points where the true relative error slightly exceeds this threshold (the values appear lower than 15\%, however). This means that the error indicator $\epsilon_{s}$ can occasionally slightly underestimate the true relative error. The choice of a small tolerance threshold $\mathcal{E}$ appears however completely relevant for accurately predicting the FRF of the structure over the whole frequency band.

The question may arise whether the adaptive refinement procedure is really needed. To address this question, the relative error made by simply interpolating the WFE solution at the coarse angular frequencies $\left\{\Omega_{p}\right\}_{p}-$ i.e., without considering any adaptive refinement - is calculated (Fig. 7). As expected, the resulting relative error presents large values over the whole frequency band. This clearly highlights the relevance of the proposed approach.
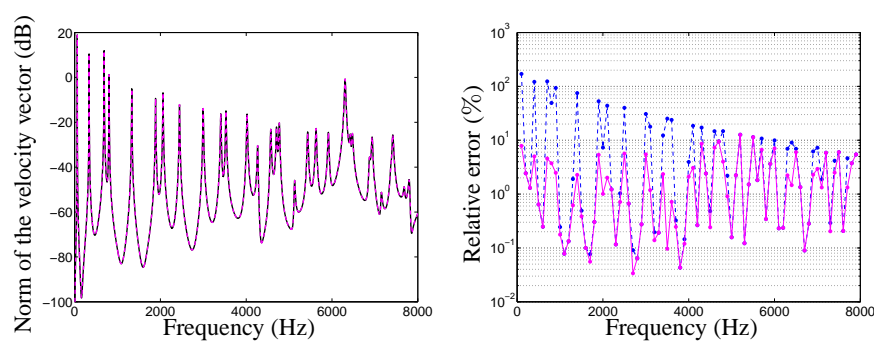

Fig. 7 FRF of the beam with holes: (Left figure) FE solution (and WFE-based interpolated solution (- - ); (Right figure) relative error of the WFE-based interpolated solution, with adaptive refinement $(-\bullet-)$ and without adaptive refinement $(-\bullet-)$.

In terms of CPU times, it takes $350 \mathrm{~s}$ to compute the forced response of the structure by means of the WFE-based interpolation strategy. This means a reduction of $60 \%$ in comparison with the conventional WFE method, and $83 \%$ in comparison with the FE method. Thus, it appears that the proposed interpolation strategy constitutes an efficient numerical tool to compute the forced response of complex periodic structures at a very small numerical cost.

For the sake of clarity, the CPU times involved by the FE method, the WFE method and the WFE-based interpolation strategy are listed in Table 1.
Table 1 CPU times involved.

\begin{tabular}{ccc}
\hline Approach used & CPU time & CPU time saving \\
\hline FE method & $2065 \mathrm{~s}$ & \\
WFE method & $868 \mathrm{~s}$ & $58 \%$ \\
Interpolation strategy & $350 \mathrm{~s}$ & $83 \%$ \\
\hline
\end{tabular}

\subsection{Curved stiffened panel}

\subsubsection{Problem description}

The dynamic behavior of a curved stiffened panel is analyzed. The panel has a constant curvature (radius of $1 \mathrm{~m}$ ), a length of $4.5 \mathrm{~m}$, and contains a periodic distribution of flat stiffeners (height of $0.05 \mathrm{~m}$ ) along both longitudinal and circumferential directions (Fig. 8). The panel and the stiffeners have the same thickness, i.e. $3 \times 10^{-3} \mathrm{~m}$. Also, they share the same material characteristics, i.e.: density $7800 \mathrm{~kg} / \mathrm{m}^{3}$, Young's modulus $210 \times 10^{9} \mathrm{~Pa}$, Poisson's ratio 0.3 , loss factor $\eta=5 \times 10^{-3}$. The periodic structure is composed of $N=15$ similar substructures, each of these being made up of seven longitudinal stiffeners and one circumferential stiffener (Fig. 9). The periodic structure is free over its right end, and subject to two point forces of same magnitude, in horizontal and vertical directions, at the top corner of its left end (Fig. 8).

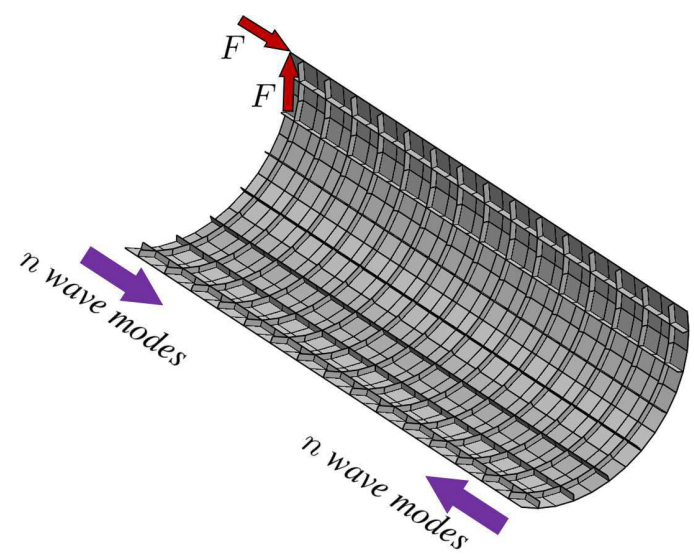

Fig. 8 Curved stiffened panel.

Each substructure is meshed by means of 2D triangular flat shell elements with three nodes and six DOFs per node that incorporate bending actions [2] and membrane actions with drilling DOFs [1]. Each substructure is discretized by means of $n=132$ DOFs on each left/right boundary, and 516 internal DOFs (Fig. 9). The dynamic behavior of the periodic structure is analyzed over the frequency band $[1 \mathrm{~Hz}, 200 \mathrm{~Hz}]$ by considering 3981 discrete 


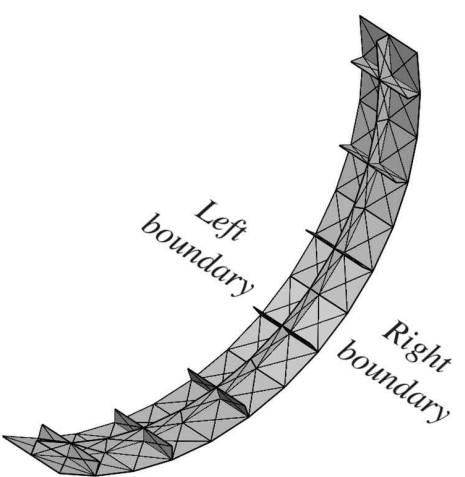

Fig. 9 FE model of a substructure (curved stiffened panel).

frequencies that are equally spaced with a step of $0.05 \mathrm{~Hz}$. Again, the CB method is used - here, 10 fixed interface modes are considered - to compute the condensed dynamic stiffness matrix $\mathbf{D}^{*}$ for each discrete frequency involved (Section 2.1.2). The WFE strategies proposed in Sections 2 and 3 are used to assess the norm of the velocity vector $\left\|i \omega \mathcal{L} \mathbf{q}^{(6)}\right\|$, i.e., on the substructure boundary 6 which is $1.5 \mathrm{~m}$ far from the left end of the panel.

\subsubsection{WFE solution}

The WFE solution is obtained by solving the matrix equation (26) for each discrete frequency involved in $[1 \mathrm{~Hz}, 200 \mathrm{~Hz}$. In the present case, the wave-based matrix equation is expressed as

$\left[\begin{array}{cc}\mathbf{I} & \boldsymbol{\Phi}_{\mathrm{F}}^{-1} \boldsymbol{\Phi}_{\mathrm{F}}^{\star} \boldsymbol{\mu}^{N} \\ \boldsymbol{\Phi}_{\mathrm{F}}^{\star} \boldsymbol{\Phi}_{\mathrm{F}} \boldsymbol{\mu}^{N} & \mathbf{I}\end{array}\right]\left[\begin{array}{c}\mathbf{Q} \\ \mathbf{Q}^{\star}\end{array}\right]=\left[\begin{array}{c}-\mathbf{F}_{0} \\ \mathbf{0}\end{array}\right]$.

The FRFs issued from the WFE and FE methods are shown in Fig. 10, along with the relative error (38). Again, the maximum values of the relative error, over small frequency bands of length $5 \mathrm{~Hz}$, are plotted. In this case again, the WFE solution perfectly agrees with the FE solution over the whole frequency band. Indeed, the relative error between the WFE and FE solutions appears very small, i.e., around $0.01 \%$. In terms of CPU times, it takes $1135 \mathrm{~s}$ to compute the forced response of the structure with the WFE method, against $3838 s$ with the FE method. This yields a reduction of $70 \%$ in benefit of the WFE method.

\subsubsection{WFE-based interpolated solution}

To reduce further the CPU time involved by the WFE method, the interpolation strategy proposed in Section 3 is used. Again, a coarse set of discrete angular frequencies $\left\{\Omega_{p}\right\}_{p}$ is chosen with an angular frequency step $\Delta \Omega$ that is ten times larger than the one used previously (i.e., $\Delta \Omega=0.5 H z$ ). Intermediate points are considered which are equally spaced,
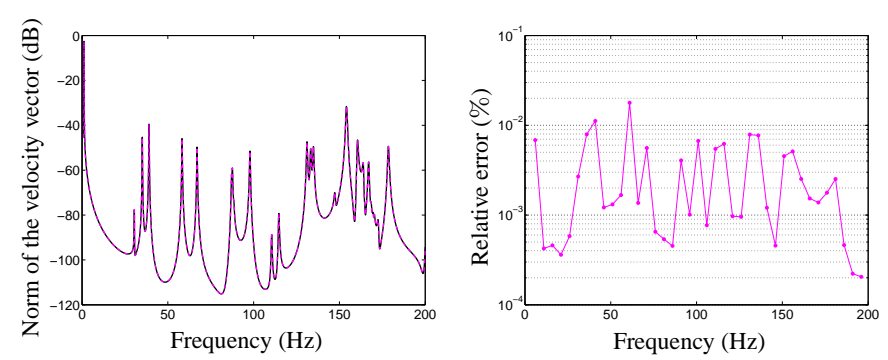

Fig. 10 FRF of the curved stiffened panel: (Left figure) FE solution (- ) and WFE solution (- - -); (Right figure) relative error of the WFE solution.

with a step of $0.05 \mathrm{~Hz}$, between two consecutive interpolation points $\Omega_{p}$ and $\Omega_{p+1}$. The adaptive refinement procedure proposed in Section 3.3 is used by considering a tolerance threshold $\mathcal{E}=10 \%$. The WFE-based interpolated solution is displayed in Fig. 11, along with the true relative error (Eq. (39)). In this case again, the interpolated FRF perfectly matches the FE solution. As expected, the true relative error appears bounded by $\mathcal{E}=10 \%$ over the whole frequency band.

Again, the relative error made by simply considering the coarse set of discrete angular frequencies $\left\{\Omega_{p}\right\}_{p}$ as interpolation points - i.e., without considering any adaptive refinement - is calculated and plotted (Fig. 11). As expected, the . predicted solution is subject to a large relative error, hence giving credit to the proposed interpolation strategy.
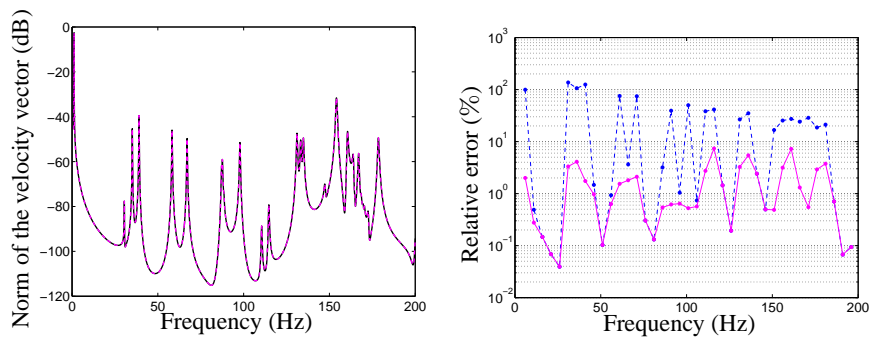

Fig. 11 FRF of the curved stiffened panel: (Left figure) FE solution $(-)$ and WFE-based interpolated solution (- - -); (Right figure) relative error of the WFE-based interpolated solution, with adaptive refinement (-๑) and without adaptive refinement (- $\bullet-)$.

In terms of CPU times, it takes $404 s$ to compute the forced response of the structure by means of the WFE-based interpolation strategy. The related CPU time saving is $64 \%$ in comparison with the conventional WFE method, and $90 \%$ in comparison with the FE method (Table 2).

Again, the relevance of the interpolation strategy, in terms of accuracy and CPU time savings, is clearly highlighted. 
Table 2 CPU times involved.

\begin{tabular}{ccc}
\hline Approach used & CPU time & CPU time saving \\
\hline FE method & $3838 s$ & \\
WFE method & $1135 s$ & $70 \%$ \\
Interpolation strategy & $404 s$ & $90 \%$ \\
\hline
\end{tabular}

\section{Conclusions}

The WFE method has been applied to compute the forced response of one-dimensional periodic structures. Some rules of thumb of the method have been highlighted and discussed. One requirement is that the substructures are to be symmetric with respect to their mid-plane. In this sense, an analytical relation can be considered to strictly enforce the coherence between the right-going and left-going wave modes. The WFE method has proved to be accurate to describe the FRFs of two sophisticated periodic structures over wide frequency bands. To reduce further the CPU times involved by the WFE method, an interpolation strategy has been proposed. In this framework, the WFE solution is explicitly computed at a reduced number of discrete frequencies. A linear interpolation scheme is then considered to approximate the WFE solution between these discrete frequencies, at several intermediate points. An error indicator has been derived which enables a fast estimation of the accuracy of the interpolation scheme at each intermediate point. Adaptive refinement of the sampling of interpolation points is achieved when the proposed error indicator exceeds a certain tolerance threshold. The relevance of the WFE-based interpolation strategy, in terms of accuracy and CPU time savings, has been clearly established in comparison with the FE method.

\section{References}

1. Allman, D.J.: Implementation of a flat facet shell finite element for applications in structural dynamics. Computers and Structures 59(4), 657-663 (1996)

2. Argyris, J.H., Papadrakakis, M., Apostolopoulou, C., Koutsourelakis, S.: The TRIC shell element: theoretical and numerical investigation. Computer Methods in Applied Mechanics and Engineering 182, 217-245 (2000)

3. Craig, R.R., Bampton, M.C.C.: Coupling of substructures for dynamic analyses. AIAA Journal 6(7), 1313-1319 (1968)

4. Duhamel, D., Mace, B., Brennan, M.J.: Finite element analysis of the vibrations of waveguides and periodic structures. Journal of Sound and Vibration 294, 205-220 (2006)

5. Gavric, L.: Computation of propagative waves in free rail using a finite element technique. Journal of Sound and Vibration 185(3), 531-543 (1995)

6. Gry, L., Gontier, C.: Dynamic modelling of railway track: a periodic model based on a generalized beam formulation. Journal of Sound and Vibration 199(4), 531-558 (1997)

7. Hetmaniuk, U., Tezaur, R., Farhat, C.: An adaptive scheme for a class of interpolatory model reduction methods for frequency response problems. International Journal for Numerical Methods in Engineering 93, 1109-1124 (2013)
8. Hussein, M.: Reduced Bloch mode expansion for periodic media band structure calculations. Proceedings of the Royal Society A 465, 2825-2848 (2009)

9. Mace, B., Duhamel, D., Brennan, M., Hinke, L.: Finite element prediction of wave motion in structural waveguides. Journal of the Acoustical Society of America 117, 2835 (2005)

10. Mace, B., Manconi, E.: Modelling wave propagation in twodimensional structures using finite element analysis. Journal of Sound and Vibration 318(4-5), 884-902 (2008)

11. Manconi, E., Mace, B., Gaziera, R.: Wave finite element analysis of fluid-filled pipes. Proceedings of NOVEM 2009 "Noise and Vibration: Emerging Methods", Oxford, UK (2009)

12. Mead, D.: A general theory of harmonic wave propagation in linear periodic systems with multiple coupling. Journal of Sound and Vibration 27(2), 235-260 (1973)

13. Mencik, J.M.: On the low- and mid-frequency forced response of elastic systems using wave finite elements with one-dimensional propagation. Computers and Structures 88(11-12), 674-689 (2010)

14. Mencik, J.M.: A wave finite element based formulation for computing the forced response of structures involving rectangular flat shells. International Journal for Numerical Methods in Engineering 95(2), 91-120 (2013)

15. Mencik, J.M., Ichchou, M.N.: Multi-mode propagation and diffusion in structures through finite elements. European Journal of Mechanics - A/Solids 24(5), 877-898 (2005)

16. Mencik, J.M., Ichchou, M.N.: Wave finite elements in guided elastodynamics with internal fluid. International Journal of Solids and Structures 44, 2148-2167 (2007)

17. Nilsson, C.M., Finnveden, S.: Waves in thin-walled fluid-filled ducts with arbitrary cross-sections. Journal of Sound and Vibration 310(1-2), 58-76 (2008)

18. Renno, J., Mace, B.: On the forced response of waveguides using the wave and finite element method. Journal of Sound and Vibration 329(26), 5474-5488 (2010)

19. Salimbahrami, B., Lohmann, B.: Order reduction of large scale second-order systems using Krylov subspace methods. Linear Algebra and its Applications 415, 385-405 (2006)

20. Signorelli, J., von Flotow, A.: Wave propagation, power flow, and resonance in a truss beam. Journal of Sound and Vibration 126(1), 127-144 (1988)

21. Waki, Y., Mace, B., Brennan, M.: Numerical issues concerning the wave and finite element method for free and forced vibrations of waveguides. Journal of Sound and Vibration 327(1-2), 92-108 (2009)

22. Z. Bai, P.D., Freund, R.: Reduced-order modeling. Handbook of Numerical Analysis Vol. XIII, Numerical Methods in Electromagnetics, W. Schilders and E.J.W. ter Maten, eds. pp. 825-895 (2005)

23. Zhong, W.X., Williams, F.W.: On the direct solution of wave propagation for repetitive structures. Journal of Sound and Vibration 181(3), 485-501 (1995) 